1 Secretaria de Saúde do Estado da Bahia (Sesab),

Centro de Endocrinologia e Diabetes do Estado da Bahia (Cedeba) - Salvador (BA), Brasil.

Orcid: https://orcid org/0000-0001-6787-

7315

mdp.dacal@gmail.com

2 Universidade do

Estado da Bahia (Uneb),

Programa de Residência

Multiprofissional em Saúde

- Salvador (BA), Brasil.

Orcid: https://orcid

org/0000-0002-8094-

2457

nysantos.010@hotmail.com

\section{Impactos das práticas integrativas e complementares na saúde de pacientes crônicos}

\author{
Impacts of integrative and complementary practices on the health of \\ chronic patients
}

Maria del Pilar Ogando Dacal'1, Irani Santos Silva $\mathbf{2}$

DOI: $10.1590 / 0103-1104201811815$

RESUMO A inclusão das práticas integrativas e complementares nos serviços de saúde pública tem acontecido de forma gradual e lentificada, há cerca de 10 anos. Este estudo visa a colaborar com a ampliação do conhecimento sobre as práticas integrativas, tendo como objetivo principal apresentar os impactos do reiki e da reflexologia podal na saúde de pacientes crônicos atendidos em um centro especializado em endocrinopatias, localizado em Salvador (BA). Trata-se de um estudo observacional retrospectivo, de corte transversal, que utilizou registros em prontuários médicos. Observaram-se, a partir da análise dos dados, impactos aparentes das terapias complementares no alívio de sintomas psicológicos, emocionais e físicos, tais como ansiedade, estresse e dores no corpo.

PALAVRAS-CHAVE Terapias complementares. Saúde pública. Doenças do sistema endócrino.

ABSTRACT The inclusion of integrative and complementary practices in public health services has been gradual and slow, about 10 years ago. This study aims to contribute to the expansion of knowledge about integrative practices, with the main objective of presenting the impacts of reiki and foot reflexology on the health of chronic patients attended at a center specialized in endocrinopathies, located in Salvador (BA). This is a cross-sectional retrospective observational study, that used records in medical records. From the data analysis, apparent impacts of complementary therapies on the relief of psychological, emotional and physical symptoms, such as anxiety, stress and body aches, were observed.

KEYWORDS Complementary therapies. Public health. Endocrine system diseases. 


\section{Introdução}

Sabe-se que o Sistema Único de Saúde (SUS), instituído pela Constituição Federal de 1988, representou um grande avanço na história brasileira. A saúde passou a ser considerada um direito de todos os cidadãos e a ser assegurada pelo Estado. Porém, nem sempre é fácil garantir o acesso igualitário às ações e aos serviços de saúde, pois há a necessidade de regulamentações por atos do legislativo, numa sociedade marcada por políticas que sempre favoreceram as elites dominantes e que visam a lucrar com o sistema de saúde brasileiro $\mathbf{}$.

Um outro desafio é a própria transformação necessária do modelo assistencial, caracterizado predominantemente pela assistência médica individual, curativa, biologicista, fragmentada e hospitalocêntrica, contrário à atenção integral à saúde'. A perspectiva da integralidade, por sua vez, fundamenta-se em uma visão holística de homem baseada no modelo biopsicossocial; na garantia de comunicação e de acesso aos diferentes níveis de atenção à saúde; na cooperação dos diferentes saberes em equipes multiprofissionais e no foco em ações de promoção da saúde e prevenção das doenças, e não apenas na assistência ${ }^{2}$.

Apesar das dificuldades, o SUS vem se sustentando no País como um processo social em permanente construção. Como afirmam Azevedo e Pelicioni ${ }^{3}$, tem ocorrido um movimento de inserção de outras racionalidades e saberes médicos no SUS, que foram legitimados a partir da homologação da Política Nacional de Práticas Integrativas e Complementares (PNPIC) pelo Ministério da Saúde, em 2006. O campo dessas práticas contempla sistemas médicos complexos e recursos terapêuticos, também denominados Medicina Tradicional e Complementar/ Alternativa (MT/MCA) 4 pela Organização Mundial da Saúde (OMS).

A Política Nacional de Práticas Integrativas e Complementares (PNPIC) contribui para o fortalecimento do SUS ao atuar nos campos da prevenção, promoção, manutenção e recuperação da saúde, sendo baseada em um modelo de atenção humanizada e centrada na integralidade do indivíduo. Além disso, representa um avanço no processo de construção do SUS, garantindo o acesso dos cidadãos brasileiros a serviços antes restritos a práticas de cunho privado 4 .

A PNPIC abarcou, inicialmente, as práticas no âmbito da Medicina Tradicional Chinesa - (Acupuntura), da Homeopatia, da Fitoterapia, da Medicina Antroposófica e do Termalismo (Crenoterapia) ${ }^{4}$. Em resposta à demanda de municípios brasileiros, o Ministério da Saúde publicou a Portaria ${ }^{\circ}$ 849 , de 23 de março de 2017, que incluiu novos procedimentos às práticas já regulamentadas pela Política: Arteterapia, Ayurveda, Biodança, Dança Circular, Meditação, Musicoterapia, Naturopatia, Osteopatia, Quiropraxia, Reflexoterapia, Reiki, Shantala, Terapia Comunitária Integrativa e Yoga ${ }^{5}$.

Posteriormente, a PNPIC foi mais uma vez atualizada a partir da publicação de nova portaria (Portaria $\mathrm{n}^{0} 702$, de 21 de março de 2018), que ampliou a oferta com a inclusão de dez práticas: apiterapia, aromaterapia, bioenergética, constelação familiar, cromoterapia, geoterapia, hipnoterapia, imposição de mãos, ozonioterapia e terapia de florais ${ }^{6}$. Dessa forma, o SUS autoriza, atualmente, 29 práticas integrativas e complementares, intensificando o desafio da capacitação, implantação e oferta destas na saúde pública do País.

Contudo, a inclusão das Práticas Integrativas e Complementares em Saúde (Pics) no SUS tem acontecido de forma gradual e muito lentificada $^{3}$. Alguns motivos considerados pelas autoras são o pouco conhecimento sobre essas terapias, a falta de pesquisas na área, além de uma ausência de profissionais qualificados na rede SUS para realizá-las. A própria OMS estimula estudos científicos para melhor conhecimento da segurança, eficácia e qualidade dessas técnicas ${ }^{4}$.

Dados coletados a partir do sistema informatizado e-SUS e do Prontuário Eletrônico 
do Cidadão (PEC) indicam que há um crescimento da procura e do acesso dos usuários do SUS às práticas integrativas. Foram registrados, em 2016, mais de 2 milhões de atendimentos com Pics realizados nas Unidades Básicas de Saúde (UBS), dos quais, mais de 770 mil foram de Medicina Tradicional Chinesa, que inclui a acupuntura; 85 mil foram de fitoterapia; e 13 mil de homeopatia. E, ainda, mais de 926 mil foram de outras práticas integrativas que não tinham um código próprio para registro e que passaram a ter com a publicação da nova portaria ${ }^{7}$.

Os dados revelam, também, que, em 2016, mais de 1.708 municípios ofereciam Pics e que $78 \%$ da distribuição dos serviços estavam concentrados na atenção básica, $18 \%$ na atenção especializada e $4 \%$ na atenção hospitalar. Ao todo, mais de 7.700 estabelecimentos de saúde ofertavam alguma prática integrativa e complementar em saúde, o que representa cerca de $28 \%$ das Unidades Básicas de Saúde (UBS). De acordo com esses dados, as Pics estão presentes em quase $30 \%$ dos municípios brasileiros, distribuídos pelos 27 estados e Distrito Federal, e em $100 \%$ das capitais brasileiras ${ }^{7}$.

No entanto, esse crescimento não tem acontecido de forma igualitária no País. Por exemplo, algumas cidades e estados têm adotado ou instituído uma Política Municipal e/ou Estadual de Práticas Integrativas, como é o caso das cidades de Recife (PE), Rio Grande do Sul (RS), Uberlândia (MG), São João Del Rei (MG), Guarulhos (SP) e dos estados do Espírito Santo, Ceará, Minas Gerais, Pará, Rio de Janeiro e Santa Catarina ${ }^{8}$. Algo que ainda não aconteceu em muitos outros estados e cidades, principalmente das regiões Norte e Nordeste.

$\mathrm{Na}$ Bahia, ainda está se discutindo, há mais de um ano, a elaboração de uma Política Estadual de Práticas Integrativas e Complementares (Pepic-BA), a partir de uma Comissão Interinstitucional de Implementação da Pepic no SUS, designada pela Portaria SES n ${ }^{0}$ 521/2016. A política tem o propósito de contribuir para a resolubilidade do sistema e para a ampliação do acesso a essas práticas nos municípios baianos, no âmbito do SUS .

Sendo assim, nota-se a importância de estudos e publicações na área das Pics na direção do seu crescimento e da sua legitimidade, através do maior conhecimento sobre a eficácia e a qualidade das práticas, com o intuito de fortalecer um movimento de expansão nacional mais uniforme quanto ao acesso e às garantias de direito ao cidadão.

\section{Práticas Integrativas e Complementares em Saúde (Pics)}

As Práticas Integrativas e Complementares tem suas bases nos sistemas médicos tradicionais, que se utilizam do modelo holístico, cujo tratamento tem o objetivo de induzir a um estado de harmonia e equilíbrio em todo organismo.

Para os antigos sistemas médicos, oriundos das tradições da China, da Índia e do Tibete, a saúde é uma questão de equilíbrio e harmonia. Eles tratam o ser humano em todos os níveis: físico, mental, emocional e espiritual. É uma questão de harmonizar o ser humano com seu meio ambiente 10(106-107).

Esse paradigma defende que um complexo sistema de fluxos de energia sutil permeia o universo"11. A energia é considerada a base de toda a vida e um fator de fundamental importância para a cura. A saúde, portanto, é concebida como um fluxo harmonioso dessas energias, e, quando em desequilíbrio, o corpo não funciona de forma eficiente. As disfunções no corpo humano são entendidas como efeitos negativos das emoções, das atitudes, do estresse, do modo de vida inadequado e dos maus hábitos alimentares.

As mesmas autoras destacam que, no Ocidente, há um demasiado enfoque no 
método científico, que excluiu, durante muito tempo, muitas técnicas curativas, com milhares de anos de aplicação bem-sucedida no Oriente. Miller ${ }^{10}$ considera que o sistema médico ocidental tende a tratar os sintomas e não o corpo inteiro, contrariamente ao sistema médico oriental, que busca tratar o ser humano em sua totalidade.

Do mesmo modo, há uma tendência de separar espírito e corpo, quando, na verdade, um não pode existir sem o outro. Para Miller10, ignorar nosso corpo ou ignorar nosso espírito não é um caminho viável. Dougans e Ellis"1(19) afirmam que "o desequilíbrio nas esferas mental e espiritual não pode ser separado do desequilíbrio físico, tamanha a complexidade dos laços que os unem".

No entanto, Miller ${ }^{10}$ considera que todos os sistemas médicos têm vantagens e inconveniências, e que o importante é como nós os utilizamos. Há uma falta de comunicação entre os diferentes sistemas e seus praticantes, que tendem a supervalorizar o seu conhecimento e negligenciar o outro.

Dougans e Ellis"1 observam que nenhuma terapia é capaz de tratar todas as doenças. A medicina complementar, assim como a medicina ocidental, deve ter o seu espaço na assistência médica, visto que ambas têm por objetivo favorecer a saúde e contribuir para o bem-estar do indivíduo.

É partindo de tal compreensão que o presente estudo tem o interesse de verificar se o uso complementar de práticas como o reiki e a reflexologia podal pode impactar e favorecer a saúde de portadores de endocrinopatias já tratadas pela medicina ocidental em um centro especializado, como um caminho norteador para potencializar a assistência prestada a esses pacientes.

A reflexologia podal é uma arte curativa respeitada e eficaz, praticada por diversas culturas ao longo da histórian". Trata-se de uma massagem terapêutica nos pés, que não deve ser confundida com a massagem básica dos pés ou com a massagem do corpo, sendo uma técnica específica de pressão que atua em pontos reflexos correspondentes a todas as partes do corpo.

Nessa perspectiva, todos os órgãos, glândulas e outras partes do organismo estão dispostos num arranjo similar ao dos pés. A reflexologia podal provoca mudanças fisiológicas no corpo, estimulando o próprio potencial de cura do organismo através da pressão, desempenhando um papel importante para a recuperação e a manutenção da saúde. Essa técnica remove os bloqueios energéticos e favorece a circulação de energia curativa em todas as partes do corpo ${ }^{11}$.

O reiki, por sua vez, segundo Miller'10, é uma técnica de cura pela imposição das mãos. Foi descoberto no fim do século XIX pelo doutor japonês Mikao Usui. O método é fundamentado na transferência de energia, de forma que o praticante de Reiki serve de condutor da energia universal (Rei) para outra pessoa, ampliando sua energia vital (Ki). A energia universal (Rei) é captada e apropriadamente transmutada para ser usada pelo receptor.

$O$ reiki visa a harmonizar o corpo e restabelecer o equilíbrio ${ }^{10}$. Recomenda-se o uso do Reiki não apenas para tratar enfermidades, mas também em caráter preventivo. Segundo De Carli12 , a terapia, ao ampliar a energia $(K i)$ que circula naturalmente pelo organismo, aumenta e mantém a saúde e a vitalidade, atuando nos bloqueios energéticos, renovando as células e promovendo saúde.

Diante do panorama exposto, este estudo visa a colaborar para a ampliação do conhecimento sobre as práticas integrativas e complementares e tem como objetivo principal apresentar os impactos possíveis do reiki e da reflexologia podal na saúde de pacientes crônicos portadores de patologias endócrinas de um centro especializado no âmbito do Sistema Único de Saúde da Bahia.

\section{Metodologia}

Este artigo refere-se a um estudo observacional retrospectivo, de corte transversal, desenvolvido no ambulatório de práticas 
integrativas e complementares de um centro especializado em diabetes e outras endocrinopatias do SUS, localizado em Salvador (BA), no período de novembro de 2016 a novembro de 2017. O ambulatório de Pics funciona desde outubro de 2015, envolvendo uma equipe multiprofissional ( 1 médica, 1 enfermeira, 1 psicóloga, 2 técnicas de enfermagem e 1 estagiária de psicologia) do referido centro e voluntários advindos de uma outra unidade de saúde do SUS parceira, na qual tais práticas já eram oferecidas há cerca de 6 anos. $\mathrm{O}$ atendimento é exclusivamente voltado para pacientes matriculados na unidade e funciona semanalmente, apenas em um turno da semana. Até o momento, as práticas oferecidas no centro especializado são reflexologia podal e reiki. Deve-se ressaltar que a escolha por ofertar tais práticas relaciona-se à qualificação dos profissionais envolvidos com a implantação do ambulatório.

A metodologia empregada neste estudo foi quantitativa, aplicada por meio de questionário estruturado, tido como protocolo de atendimento pela equipe responsável após um ano de funcionamento do ambulatório e anexado ao prontuário dos usuários. Nesse questionário, apresentam-se dados sobre o perfil dos pacientes (idade, tempo na unidade, patologia de base, forma de encaminhamento para o ambulatório); queixas iniciais; modalidades de atendimento em Pics a que se submeteu; frequência no ambulatório; e percepção de melhora dos sintomas numa escala de intensidade de 0 a $100 \%$. Os dados foram tabulados, tratados estatisticamente e apresentados por meio de porcentagem simples, utilizando o programa Microsoft Excel ${ }^{\circledR} 2010$.

Deve-se ressaltar que o estudo observou e cumpriu os princípios éticos para a publicação de artigos com resultados envolvendo seres humanos segundo a declaração de Helsinki, sendo aprovado pelo Comitê de Ética em Pesquisa (CEP) da Secretaria da Saúde do Estado da Bahia (Sesab), CAAE ${ }^{\circ}$ 71345317.7.0000.0052.

\section{Resultados}

Encontrou-se um quantitativo de 244 pacientes que foram atendidos com práticas integrativas e complementares no ambulatório ao longo de um ano de funcionamento. Foram realizados 453 atendimentos de reiki e 419 atendimentos de reflexologia podal, perfazendo um total de 872 atendimentos.

Para o estudo em questão, foram coletados dados de 59 pacientes, sendo eles 55 mulheres e 4 homens, com idade média de 53,2 anos e com um tempo médio de 7,6 anos de tratamento endócrino na unidade. Com relação à patologia de base: $53 \%$ dos pacientes tinham obesidade; $27 \%$ eram portadores de diabetes; e $20 \%$ apresentavam doenças da tireoide.

$\mathrm{O}$ acesso às práticas ofertadas pelo ambulatório de Pics ocorreu por encaminhamento de profissionais diversos da unidade de saúde referida ou por demanda espontânea. Os encaminhamentos, em sua maioria, foram feitos por psicólogos (36\%), seguidos por: demanda espontânea (27\%), médicos (15\%), nutricionistas (10\%), enfermeiros $(5 \%)$, assistentes sociais (3\%) e fisioterapeutas (3\%). Vale destacar, portanto, o interesse e a procura por parte dos usuários, uma vez que o percentual de demanda espontânea se apresenta superior aos encaminhamentos dos demais profissionais de saúde, com exceção da categoria dos psicólogos.

Das Pics oferecidas, aproximadamente $70 \%$ dos pacientes foram atendidos com ambas as terapias (reiki e reflexologia podal), $15 \%$ apenas reiki e outros $15 \%$ apenas reflexologia podal. A maioria dos pacientes $(69 \%)$ realizou até 5 sessões, $29 \%$ dos pacientes realizaram entre 6 e 10 sessões, $2 \%$ realizaram acima de 15 sessões, sendo que nenhum paciente realizou entre 10 e 15 sessões. Todos os pacientes referem o desejo de continuar frequentando o ambulatório de Pics.

Quanto às principais queixas, $85 \%$ dos pacientes referiram dores no corpo, e $80 \%$ relataram ansiedade, seguida por: estresse e cansaço (ambos, 78\%); inchaço nas pernas 
e nos pés (66\%); ganho de peso (50\%); e insônia (46\%). Os pacientes ainda referiram pressão arterial elevada (44\%); depressão (39\%); constipação (31\%); glicemia alta (34\%); sintomas da menopausa (24\%); e cólicas (12\%) (gráfico 1). Tais queixas apresentam-se como motivações para os encaminhamentos por parte dos profissionais, bem como apresentam relação direta com a demanda espontânea dos pacientes para o ambulatório de Pics.

Quanto às melhoras dos sintomas percebidas pelos pacientes após acompanhamento com as Pics, tem-se que $51 \%$ dos pacientes relataram melhora nas dores no corpo e no estado de estresse, além da melhora percebida em: cansaço (39\%); ansiedade e inchaço nas pernas e nos pés (ambos, 34\%); e insônia (27\%). Os pacientes ainda perceberam melhoras em sintomas como: pressão arterial (17\%); depressão (15\%); ganho de peso (13\%); constipação (7\%); glicemia alta (7\%); cólicas (2\%); e sintomas da menopausa (2\%) (gráfico 1). Vale destacar que o maior percentual (51\%) de pacientes que relataram melhoras percebidas refere-se, também, aos maiores percentuais relativos às queixas iniciais: dores no corpo (85\%) e estresse (78\%).

Gráfico 1. Porcentagem dos pacientes que apresentaram queixas iniciais e identificaram melhora dos sintomas após acompanhamento com as Pics (100\% da amostra)

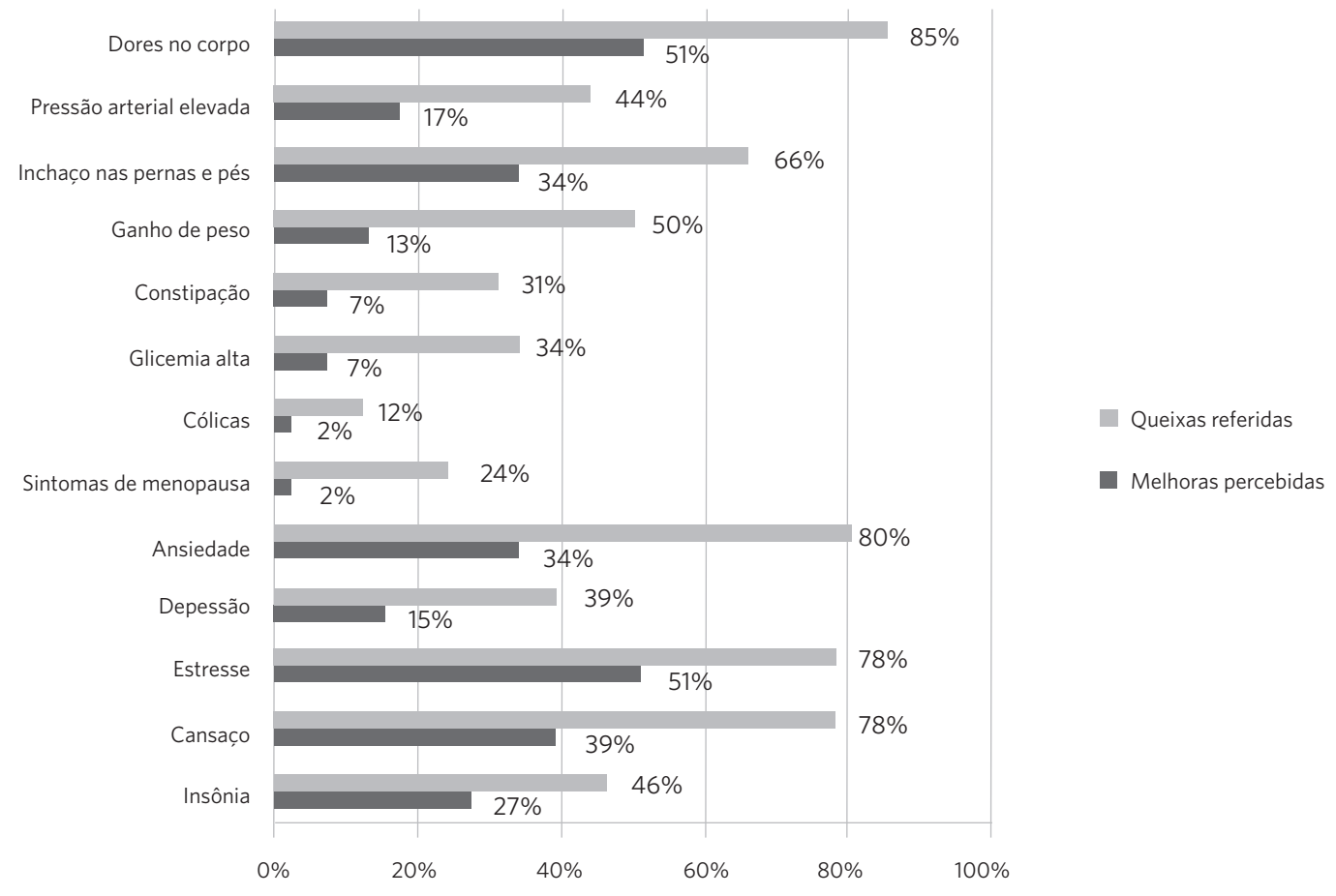

Fonte: Elaboração própria. 
Ao avaliar a percepção de melhora dos sintomas segundo o uso das práticas, não foi possível perceber diferença significativa entre o reiki e a reflexologia podal quando usadas isoladamente, mas foi identificada uma tendência a um percentual maior de melhora entre os pacientes que fizeram uso combinado destas apenas com relação aos sintomas com também maior percentual de queixa inicial (acima de 46\%): insônia, inchaço nos pés e nas pernas, estresse, ansiedade e dores no corpo.

Os pacientes também avaliaram a intensidade da redução dos sintomas, numa escala de 0 a 100\%, obtendo-se um resultado médio de $64 \%$ de intensidade de melhora quanto à queixa inicial, o que sugere um impacto importante na qualidade de vida dos participantes deste estudo. Ressalta-se que a maioria dos pacientes realizou apenas até 5 sessões de Pics (69\% dos pacientes). Comparandose os grupos de pacientes, no que diz respeito ao quantitativo de sessões realizadas, observa-se que o grupo que realizou até 5 sessões $(\mathrm{N}=41,69 \%$ da amostra) obteve um percentual médio de intensidade de melhora de $62 \%$, enquanto o grupo que realizou de 6 a 10 sessões ( $N=17,29 \%$ dos pacientes) apresentou um resultado médio na intensidade da melhora de $69 \%$. O paciente que realizou acima de 15 sessões ( $\mathrm{N}=1,2 \%$ da amostra) referiu percentual de $100 \%$ com relação à intensidade de melhora dos sintomas.

Os dados indicam que o acompanhamento com práticas integrativas e complementares favorece a redução aparente da intensidade dos sintomas com um quantitativo reduzido de sessões (5), assim como é possível observar que o prolongamento do acompanhamento tende a gerar maiores resultados, uma vez que a intensidade da melhora identificada se apresentou maior com o acréscimo de atendimentos.

Analisaram-se, também, possíveis relações entre a intensidade da melhora e a prática aplicada. Entre $15 \%$ dos pacientes atendidos apenas com reflexologia podal, foi observada uma média de $72 \%$ na intensidade da melhora identificada. Para os outros 15\% que foram atendidos apenas com reiki, a média de intensidade da melhora encontrada foi de $55 \%$. A maioria dos pacientes (70\%) foi atendida com ambas as práticas, e a média de intensidade da melhora identificada foi de $65 \%$.

Os resultados obtidos sugerem uma maior satisfação por parte dos usuários com o tratamento da reflexologia podal quando comparado com o tratamento com reiki. O que, por sua vez, pode sugerir resultados mais imediatos ou mais perceptíveis com o uso da reflexologia, ou mesmo uma melhor adequação da terapêutica às queixas iniciais mais comuns entre os pacientes. No entanto, a intensidade da melhora percebida para o tratamento combinado das duas terapias se aproximou de forma significativa do percentual de intensidade do tratamento único com reflexologia, o que pode sugerir possíveis benefícios do uso combinado de diferentes práticas.

De forma geral, neste estudo, foi possível identificar possíveis benefícios referentes ao uso da reflexologia podal e do reiki no tocante às queixas iniciais dos pacientes da amostra. No entanto, torna-se necessário destacar limitações neste estudo para fazer inferências com relação a especificidades do uso exclusivo da reflexologia podal ou do reiki, pois a maioria dos pacientes $(70 \%)$ se submeteu às duas práticas. Portanto, encorajam-se outros estudos que possam analisar com mais profundidade os efeitos dessas duas terapias complementares de forma comparativa e com uso de parâmetros biomédicos que possam ser associados ao discurso e à avaliação subjetiva dos pacientes.

\section{Discussão}

Machado ${ }^{\mathbf{3}}$, a partir de uma extensa revisão de literatura, aponta uma crescente procura de pacientes com Diabetes Mellitus (DM) por tratamentos complementares, sendo esta uma doença crônica de difícil controle e que 
acarreta diversas complicações. Esse autor sinaliza que a terapia à base de ervas tem sido o método mais comum utilizado por essa clientela, havendo outras práticas de Pics que são também usuais, como: práticas de relaxamento (ex: acupuntura, massagens, aromaterapia), cura espiritual (rezas), homeopatia, quiropraxia e suplementação nutricional.

Os achados do estudo realizado por Machado ${ }^{13}$ indicam um expressivo interesse de pais e/ou responsáveis legais de crianças e adolescentes portadoras de DM1 pela utilização de terapias complementares para esses pacientes. Uma das principais motivações foi o desejo de experimentar alternativas que ajudassem na melhora da glicemia e que diminuíssem os riscos trazidos com a evolução da doença.

No presente trabalho, também foi possível observar a procura dos usuários pelas Pics com vistas a um melhor controle glicêmico (34\%), apesar de que apenas $7 \%$ dos pacientes relataram melhora na glicemia após fazerem uso das Pics. No entanto, $51 \%$ dos pacientes perceberam melhoras nas dores no corpo e $34 \%$ referiram alívio do inchaço nas pernas e nos pés, sintomas característicos de pessoas com diabetes. Tais resultados sugerem, portanto, benefícios importantes no controle das complicações do diabetes, colocando as práticas utilizadas em um lugar de terapia complementar que pode elevar a qualidade de vida de pacientes com tal patologia.

No estudo de Machado ${ }^{\mathbf{1 3}}$, mais da metade dos pais (59\%) responderam acreditar que o uso das Pics teve eficácia, contribuindo para uma melhor qualidade de vida dos pacientes, sendo que $94 \%$ dos entrevistados referiram uma melhoria nas medições glicêmicas. No entanto, neste estudo de Machado, a melhoria da qualidade de vida foi avaliada subjetivamente pela percepção da família, assim como no presente estudo os dados analisados referem-se a avaliações subjetivas dos próprios pacientes quanto a possíveis resultados dos atendimentos com Pics.

Sendo assim, fazem-se necessários estudos que verifiquem especificamente a eficácia das Pics no controle glicêmico, bem como na prevenção e no controle das complicações do diabetes, utilizando critérios biomédicos, tais como diminuição da dose de insulina e medicações orais, ou mesmo o nível de hemoglobina glicada - HbAl. Do mesmo modo, estudos longitudinais que avaliem a associação entre o uso das terapias complementares e o aparecimento de comorbidades referentes ao diabetes também podem apresentar resultados mais específicos e mais criteriosos.

No que diz respeito ao uso de Pics no tratamento da obesidade, Ornela, Oba, Kinouchi, et al. ${ }^{14}$ realizaram estudo clínico randomizado com o objetivo de analisar o efeito da estimulação dos pontos de acupuntura. A partir do acompanhamento oferecido, observou-se uma resposta com relação à perda de peso e medidas corporais (redução de Índice de Massa Corporal - IMC - e circunferência abdominal) com 10 sessões de acupuntura, aliadas a atividade física e reeducação alimentar. O estudo destaca resultados da acupuntura não somente na redução do peso, mas, também, na melhora de aspectos da qualidade de vida dos pacientes com obesidade, tais como controle da ansiedade, tensão emocional e autoestima, além de aumentar a motivação.

No presente estudo, o ganho de peso apareceu como uma das queixas referidas pelos pacientes com significativa expressão (50\% dos pacientes), sendo que apenas $13 \%$ dos pacientes relataram redução de peso após uso das terapias complementares. No entanto, foi possível observar que $34 \%$ dos pacientes referiram redução da ansiedade, aspecto que tende a interferir no comportamento alimentar dos indivíduos. Vale destacar que, segundo Marchi-Alves, Nogueira, Mendes, et al. (2010), citado por Ornela, Oba, Kinouchi, et al. $^{14}$, o manejo da ansiedade é complexo, exigindo cuidados multiprofissionais que incluem terapêuticas medicamentosas ou não, tais como nutricionais, psicológicas e de 
reeducação de hábitos de vida. Terapêuticas essas que não foram analisadas nem controladas por este estudo.

Não foram encontrados na literatura estudos referentes ao uso das Pics no tratamento das doenças da tireoide, outra endocrinopatia presente na amostra deste estudo. A análise dos dados, no entanto, indica que esses pacientes podem se beneficiar das Pics, uma vez que entre as manifestações clínicas do hipertireoidismo e do hipotireoidismo, por exemplo, observam-se sintomas como nervosismo/irritabilidade e ansiedade ${ }^{15,16}$, sobre os quais foi observado impacto positivo após uso das Pics no presente estudo, com $34 \%$ dos pacientes sinalizando melhora dos níveis de ansiedade.

Com relação a estudos que investigam o alcance da reflexologia podal e do reiki, foi encontrado um cenário de maior carência, talvez pelo fato dessas práticas terem sido incluídas recentemente nos procedimentos da Política Nacional de Práticas Integrativas e Complementares.

No entanto, um estudo realizado por Machado, Rodrigues e Silva ${ }^{17}$ demonstrou efeito positivo da utilização da reflexologia podal na melhora da qualidade do sono, apesar das autoras estimularem outros estudos para maior entendimento e padronização dos resultados. Freitag, Andrade e Badke ${ }^{\mathbf{1 8}}$, por sua vez, a partir de uma revisão de literatura, apontaram que o uso do reiki provoca mudanças significativas na saúde do indivíduo, principalmente no que se refere a ansiedade, dores, estresse, aumento das células de defesa e diminuição dos níveis pressóricos.

No presente estudo, o uso combinado do reiki com a reflexologia podal, em $70 \%$ da amostra, evidenciou uma percepção de melhora importante do estresse e das dores no corpo, com $51 \%$ da amostra identificando tal impacto com relação a ambos os sintomas. Em seguida, ansiedade, inchaço em pés e pernas e insônia foram sintomas que aparentemente reduziram de forma expressiva, segundo a percepção dos participantes.
No que diz respeito ao uso isolado e específico do reiki ou da reflexologia podal (15\% da amostra para cada terapêutica), no entanto, a análise dos dados não evidenciou diferenças significativas entre tais práticas quanto à redução percebida dos sintomas iniciais.

Ainda que a análise dos resultados quanto ao uso isolado do reiki ou da reflexologia podal não apresente dados suficientes para sugerir terapêuticas específicas, o uso combinado aponta para repercussões importantes em sintomas iniciais, em conformidade com o evidenciado por Machado, Rodrigues e Silva ${ }^{\text {17, }}$, assim como por Freitag, Andrade e Badke ${ }^{18}$ : melhora da qualidade do sono, da ansiedade, das dores e do estresse.

De forma geral, foi possível observar no presente estudo o impacto das Pics no alívio de sintomas psicológicos, a exemplo da ansiedade; emocionais, como o estresse; e físicos, tais como dores no corpo, cansaço, inchaço nas pernas e nos pés e insônia.

Como mencionado, as Pics buscam tratar o indivíduo em sua totalidade e consideram que há uma influência mútua entre os desequilíbrios orgânicos e emocionais, o que nos direciona a uma reflexão importante a respeito de como o impacto percebido pelos pacientes desta amostra pode repercutir não somente na sua qualidade de vida, mas na adesão ao tratamento da medicina convencional, na relação subjetiva desses indivíduos com a experiência do adoecer e com seu processo saúde-doença. Aspectos esses que, por sua vez, podem vir a se desdobrar em uma melhora dos índices biomédicos que evidenciam o controle da patologia endócrina de base, reafirmando a perspectiva holística defendida pela PNPIC no campo da saúde.

\section{Conclusões}

Os achados permitem afirmar que há uma alta demanda pelas Pics por parte dos usuários e uma aparente percepção de seus benefícios. Os dados indicam impactos positivos 
na saúde dos usuários nas dimensões psicológica, física e emocional, bem como demonstram os benefícios do uso particular das Pics por pessoas com doenças crônicas, especificamente de origem endócrina. Tais patologias compõem um quadro clínico complexo, com a sobreposição de sintomas físicos/orgânicos e sintomas psicológicos, que demandam uma abordagem integral à saúde dos indivíduos.

Estudos posteriores são necessários para avaliar particularidades do impacto do uso de Pics na evolução de doenças crônicas e em aspectos específicos das endocrinopatias, com o auxílio de medidas biomédicas que possam delimitar e aprofundar o conhecimento em torno dos ganhos de complementar o tratamento convencional com o uso de outras racionalidades médicas em processo de inclusão no SUS. Do mesmo modo, estudos que discriminem e avaliem práticas integrativas e complementares específicas no âmbito da endocrinologia podem favorecer a aplicação destas de forma mais assertiva e eficaz, bem como a elaboração de protocolos e terapêuticas de forma cada vez mais individualizada.

Vale destacar a necessidade de reconhecimento e aceitação das Pics por parte dos profissionais de saúde, algo também evidenciado neste estudo, uma vez que os encaminhamentos na unidade se concentraram nos profissionais da área de psicologia e na demanda espontânea dos usuários do serviço. Tal cenário aponta para uma das dificuldades de implantação e estruturação das Pics no SUS: a legitimação de novas racionalidades médicas, já regulamentadas por uma política nacional, mas ainda com restrito espaço entre profissionais de saúde e gestores. Isso implica a necessidade de reformulações na PNPIC em direção ao fomento a pesquisa, ensino e extensão, além da participação e do controle popular na execução dessa política.

Ainda são escassos no Brasil estudos sobre os benefícios das Pics na saúde pública. Sendo assim, torna-se clara a necessidade de estudos científicos mais estruturados que se debrucem sobre aspectos como qualidade de vida, adesão ao tratamento e promoção da saúde, e que utilizem medidas mais confiáveis para avaliar os impactos das Pics na saúde dos pacientes. Isso porque evidências científicas nessa área servirão para o fortalecimento e a expansão das Pics, bem como para a ampliação do olhar sobre o adoecer e as práticas em saúde.

\section{Colaboradores}

Dacal MPO e Silva IS participaram conjuntamente da concepção, do planejamento, da análise e da interpretação dos dados, bem como da elaboração do rascunho e da revisão crítica do conteúdo. Ambas as autoras aprovaram a versão final do manuscrito e declaram ser responsáveis por todos os aspectos do trabalho, garantindo sua precisão e integridade.

\section{Agradecimentos}

Agradecemos à equipe de Pics da unidade, aos pacientes do Ambulatório de Práticas Integrativas e Complementares e a todos os voluntários que ofereceram seus serviços nessa instituição. Esperamos que esta discussão possa contribuir para a ampliação e a melhoria da oferta das Pics no nosso estado. 


\section{Referências}

1. Aguiar ZN. O Sistema Único de Saúde e as Leis Orgânicas da Saúde. In: Aguiar ZN, organizador. SUS: Sistema Único de Saúde: Antecedentes, Percurso, Perspectivas. 1. ed. São Paulo: Martinari; 2011. p. 4268.

2. Rocha SA, Bocchi SCM, Juliani CMCM. O princípio da integralidade no Sistema Único de Saúde (SUS): utopia? Rev. Inter. Interdisc INTERthesis [internet]. 2011 jan-jul [acesso em 2017 abr 22]; 8(1):120132. Disponível em: https://periodicos.ufsc.br/index.php/interthesis/article/view/14837.

3. Azevedo E, Pelicioni MCF. Práticas Integrativas e Complementares de Desafios para a Educação. Trab Educ Saúde [internet]. 2011 nov [acesso em 2017 abr 22]; 9(3):361-378. Disponível em: http://www.scielo.br/scielo.php?pid=S1981$-77462011000300002 \&$ script=sci_abstract\&tlng=pt.

4. Brasil. Ministério da Saúde. Secretaria de Atenção à Saúde. Departamento de Atenção Básica. Política Nacional de Práticas Integrativas e Complementares no SUS. Brasília, DF: MS; 2006.

5. Brasil. Ministério da Saúde. Portaria $\mathrm{n}^{0} 849$, de 27 de março de 2017. Inclui a Arteterapia, Ayurveda, Biodança, Dança Circular, Meditação, Musicoterapia, Naturopatia, Osteopatia, Quiropraxia, Reflexoterapia, Reiki, Shantala, Terapia Comunitária Integrativa e Yoga à Política Nacional de Práticas Integrativas e Complementares. Diário Oficial da União. 28 Mar 2017.

6. Brasil. Ministério da Saúde. Portaria ${ }^{\circ} 702$, de 21 de março de 2018. Altera a Portaria de Consolidação ${ }^{\circ}$ 2/GM/MS, de 28 de setembro de 2017, para incluir novas práticas na Política Nacional de Práticas Integrativas e Complementares - PNPIC. Diário Oficial da União. 22 Mar 2018.

7. Brasil. Ministério da Saúde. Secretaria de Atenção à Saúde. Departamento de Atenção Básica. Portal da saúde [internet]. Brasília, DF: MS; 2017 [acesso em 2017 jan 23]. Disponível em: http://dab.saude.gov. br/portaldab.

8. Sabedoria Política [internet]. [Sem local]: c2011 [acesso em 2017 fev 12]. Disponível em: https:// www.sabedoriapolitica.com.br.

9. Bahia. Secretaria da Saúde [internet]. Salvador: Sesab; 2016 [acesso em 2017 fev 12]. Disponível em: http://www.saude.ba.gov.br/.

10. Miller JP. O livro dos chakras, da energia e dos corpos sutis: uma nova visão das tradições antigas e modernas sobre os nossos centros de energia. 1. ed. São Paulo: Pensamento; 2015.

11. Dougans I, Ellis S. Um guia passo a passo para a aplicação da reflexologia. 9. ed. São Paulo: Cultrix; 1999.

12. De Carli J. Reiki: Amor, Saúde e Transformação. 5. ed. São Paulo: Alfabeto; 2013.

13. Machado LCB. Práticas integrativas e complementares no tratamento de crianças e adolescentes com diabetes melito tipo 1: construção de um perfil [dissertação]. Salvador: Universidade Federal da Bahia; 2012. 66 p. [acesso em 2017 abr 22]. Disponível em: http://repositorio.ufba.br/ri/handle/ri/15742.

14. Ornela RG, Oba MV, Kinouchi FL, et al. Acupuntura no tratamento da obesidade. J Health Sci Inst. 2016; 34(1):17-23.

15. Maia AL, Scheffel RS, Meyer ELS, et al. Consenso brasileiro para o diagnóstico e tratamento do hipertireoidismo: recomendações do Departamento de Tireoide da Sociedade Brasileira de Endocrinologia e Metabologia. Arq Bras Endocrinol Metab [internet]. 2013 abr [acesso em 2017 ago 11]; 57(3):205-232. Disponível em: http:// www.scielo.br/scielo.php?script=sci_arttext\&pid $=$ S0004-27302013000300006.

16. Brenta G, Vaisman M, Sgarbi JA, et al. Diretrizes clínicas práticas para o manejo do hipotiroidismo. 
Arq Bras Endocrinol Metab [internet]. 2013 jun [acesso em 2017 ago 11]; 57(4):265-299. Disponível em: http://www.scielo.br/scielo.php?pid=S0004$-27302013000400003 \&$ script=sci_arttext\&tlng=pt.

17. Machado FAV, Rodrigues CM, Silva PA. Influência da Reflexologia Podal na qualidade do sono: estudo de caso. Cad Naturol Terap Complem [internet]. 2013 [acesso em 2017 abr 22]; 2(3):67-75. Disponível em: http://portaldeperiodicos.unisul.br/index. php/CNTC/article/view/1536/1807.
18. Freitag VL, Andrade A, Badke MR. O Reiki como forma terapêutica no cuidado à saúde: uma revisão narrativa da literatura. Enfermería Global [internet]. 2015 abr [acesso em 2017 abr 22]; 38:346356. Disponível em: http://scielo.isciii.es/pdf/eg/ v14n38/pt_revision5.pdf.

Recebido em 30/04/2018

Aprovado em 16/08/2018

Conflito de interesses: inexistente

Suporte financeiro: não houve 